\title{
Experimental and Theoretical Realization of Zenneck Wave-based Non-Radiative, Non-Coupled Wireless Power Transmission
}

Sai Kiran Oruganti, ${ }^{1}$ Jagannath Malik, ${ }^{1}$ Jongwon Lee, ${ }^{1}$ Woojin Park, ${ }^{1}$ Bonyoung Lee, ${ }^{1}$ Seoktae Seo, ${ }^{1}$ Dipra Paul,${ }^{1}$ Haksun Kim, ${ }^{1}$ Thomas Thundat ${ }^{2}$ and Franklin Bien. ${ }^{1 *}$

${ }^{1}$ Ulsan National Institute of Science and Technology, Republic of Korea ${ }^{2}$ University at Buffalo, New York, USA

*To whom correspondence should be addressed; E-mail: bien@unist.ac.kr.

A decade ago, non-radiative wireless power transmission re-emerged as a promising alternative to deliver electrical power to devices where a physical wiring proved to be unfeasible. However, conventional coupling-based approaches are neither scalable nor efficient when multiple devices are involved, as they are restricted by factors like coupling and external environments. Zenneck waves are excited at interfaces, like surface plasmons and have the potential to deliver electrical power to devices placed on a conducting surface. Here, we demonstrate, efficient and long range delivery of electrical power by exciting non-radiative waves over metal surfaces to multiple loads. Our modeling and simulation using Maxwells equation with proper boundary conditions shows Zenneck type behavior for the excited waves and are in excellent agreement with experimental results. In 


\section{conclusion, we physically realize a radically different power transfer system, based on a wave, whose existence has been fiercely debated for over a century.}

\section{Keywords: Zenneck Waves, Wireless Power Transfer, Power, Internet of things, electromagnetic shielding}

In 2007, coupled WPT re-emerged as an alternative to deliver electrical power to systems where physical wiring is difficult or dangerous ${ }^{1,2}$. Since then, a number of notable articles appeared $^{3-5}$. However, these were improvements or at the best variations of the coupled WPT systems originally proposed in ${ }^{2}$.

All the existing conventional WPT systems(Inductive, magnetic resonance and capacitive) rely on critical coupling between coils of the transmitter and the receiver for efficient delivery of power. The resonance conditions are easily affected by the external factors ${ }^{6}$. It has been also well understood that the need for a critical coupling leads to peak splitting phenomena for multiple resonant devices ${ }^{7}$. This causes efficiency degradation and hence, are unsuitable for emerging fields such as, internet of things(IoT) and dynamic charging of electrical vehicles. It is therefore, Assawaworrarit et al, proposed parity time circuits to resolve the issue of dynamic wireless charging ${ }^{6}$. Unfortunately, we will continue to face these limitations as long as we rely on critical coupling.

A non-radiating wave-based wireless power transfer system would be a desirable candidate to solve these issues. Quite a few wave based systems in the $\mu$-wave regime have appeared over the years. A detailed literature survey of these systems has been carried out in the supplementary material. Also, WPT systems saw the usage of magneto-inductive planar waveguide ${ }^{8}$. This kind of WPT system utilizes the concept of meta-materials and 
generation of standing waves. One may come to a conclusion that this is the meta-material equivalent of the quarter-wave Tesla transformer.

We wish to draw the attention to Zenneck wave(Sommerfeld-Zenneck wave), which resides at the metal-air interface, akin to surface plasmons (SP) and surface waves(SW) ${ }^{9,10}$. While SP and surface wave (SW) have been widely researched areas in optical physics and metasurfaces, they are relatively less studied in the microwave regime ${ }^{11,13-15}$. Likewise, much research around ZW is focused on the communications and geophysics applications $^{16-18}$. Unfortunately, ZW has been surrounded by the controversies regarding their physical existence ${ }^{20-22}$. The bulk of the controversy arose from the alleged sign error committed by Sommerfeld in $1909^{21,22}$. Some authors have shown feasibility of such waves by recreating the critical Seneca lake experiment to debunk the Sommerfeld sign error $\operatorname{myth}^{23}$. Quite literally, one does not find any study on the utilization of ZW for nonradiative power transfer. Recently in 2014 and 2017 Sarkar et al, have taken great pains to clarify the confusions arising due to the definitions of SW, SP and ZW through their mathematically rigorous articles ${ }^{21,22}$. The properties exhibited by ZWs are like SW and SP, with certain differences. All these three physical phenomena are transverse magnetic (TM) modes and exhibit evanescent field decay away from the metal-air or metal-dielectric or conductive-dielectric interface. Unlike SW, the ZW come into existence as a result of zero of the TM reflection coefficient. SP come into existence at the quasi-particle levels. Whereas, ZW propagate in the form of localized charge oscillations. Just like SW and SP, when ZW are excited on metal surfaces, the net flow of current is zero. The Brewster angle of incidence in case of ZW is frequency independent. Therefore, the attenuation of $\mathrm{ZW}$ waves is frequency independent and the attenuation rate is slow in the transverse direction ${ }^{21,22}$. They sink into a lossy dielectric media, as mathematically demonstrated by Barlow and Cullens in their classic article ${ }^{24}$. This sinking phenomenon was later ex- 
perimentally demonstrated by Jangal et al and Ling et al $^{16,25}$. Here we demonstrate the physical realization of a ZW non-radiative power transmission using the arrangement of a planar ground backed impedance (GBI) surface and a half wave helical transformer at radio frequency $(\mathrm{RF})$. The GBI structure establishes a TM wave across the metal surface, whereas, the half wave helical transformer drives the voltage across the GBI terminals. The helical transformer is like the telsa transformer (supplementary information). However, unlike the tesla transformer it does not generate standing waves. It was earlier theorized that an infinite vertical aperture is needed to excite a Zenneck wave and hence was not physically realizable ${ }^{12}$. In our results we demonstrate that, although it is not possible to excite a pure ZW, however, waves with strong ZW like properties can certainly be excited. Thus bypassing the problem of infinite vertical aperture. We also demonstrate that unlike the coupled non-radiative wireless power transmission systems, the presence of leaky metal shields does not affect the power transmission efficiency ${ }^{2,26}$. Moreover, we demonstrate uniform power delivery to multiple receiving units with meaningful efficiency by theory and experiment, as we eliminate the frequency peak splitting issue altogether ${ }^{7}$. We also demonstrate by arriving at the equation 1, that equi-phases of ZW waves tilt backwards in the air, at the metal-air interface ${ }^{9,10}$. Thus, reminiscent with the title of the article by Jeon et.al. ${ }^{17}$. This article implies that there is a link between SP and ZW's at metal-air interface.

While efficient transmission of non-radiative, wireless power over long distances using earth as a conductor is far from practical realization, it may be possible to utilize already existing metal structures to send guided mode waves for powering electrical devices ${ }^{1,27,28}$. There exist many practical scenarios consisting of metallic infrastructures, such as nuclear plants, railway tracks, space ships, steel building structures, pipelines, etc. Practical applications include powering Internet of things (IoT) devices, charging for -marine vessels, 
a

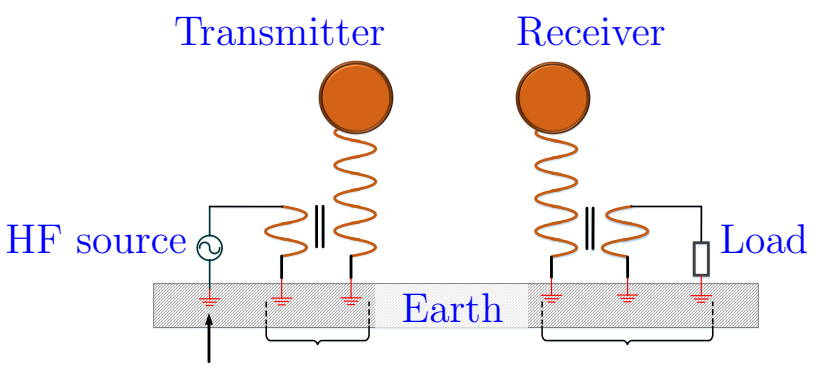

b

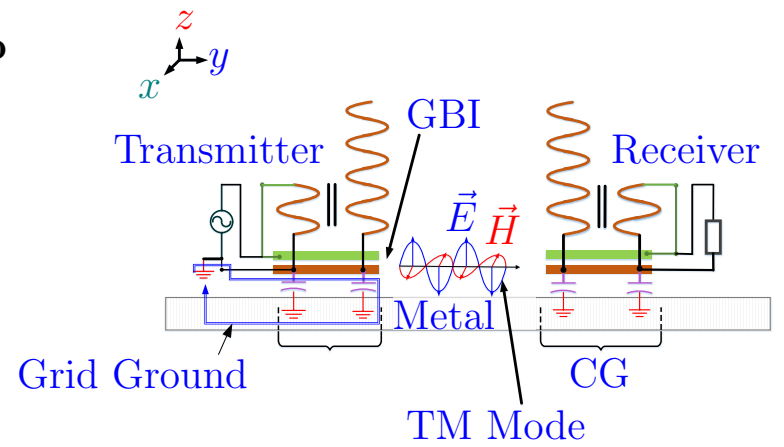

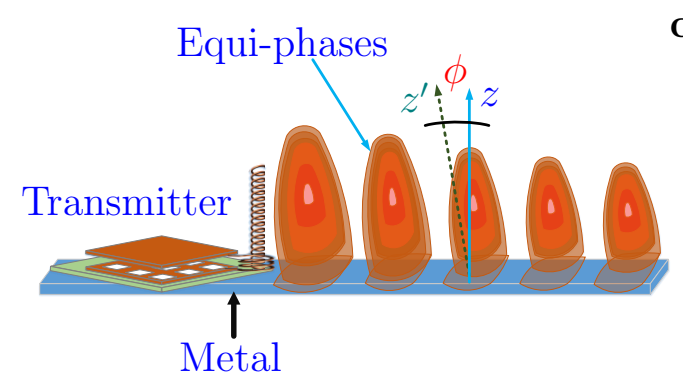

Equi-phase sinking

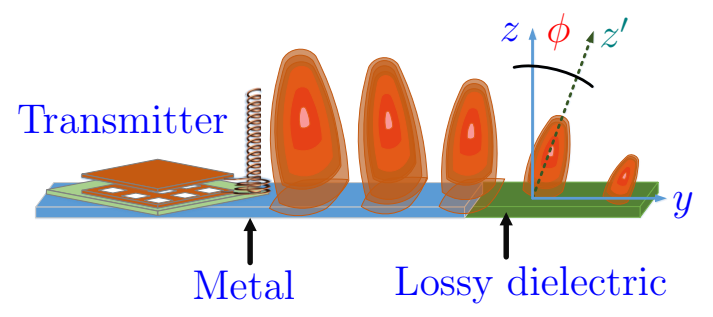

Fig. 1: Concept of the proposed Zenneck wave system (a) In Tesla transformer, grounding is an extremely critical factor. Both the transmitter and receiver are grounded to the earth ground. (b) Approach followed in this study based on half wave helical transformer. The GBI resonator sets up a TM-Mode wave. Capacitive grounding $[\mathrm{CG}]$ is done through the metal, which in turn pulls the reference potential of the metal to the grid ground.(c)Localized field oscillation forms the Equi-phases, with an angle of backward tilt $\phi$ (negative). In case of imperfect ground, this tilt is forward (d) The equi-phases undergo a "forward" tilt $(\phi)$ in a lossy dielectric and sink. 
$\mathbf{a}$

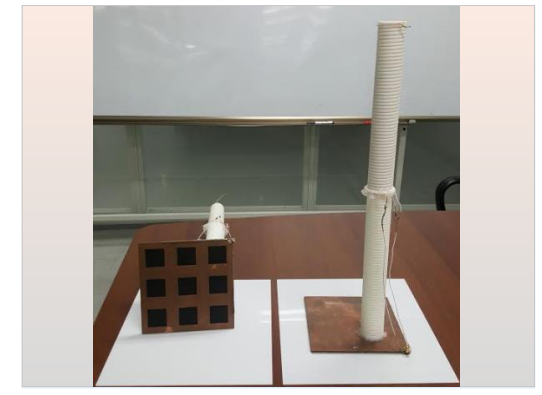

Resonator System

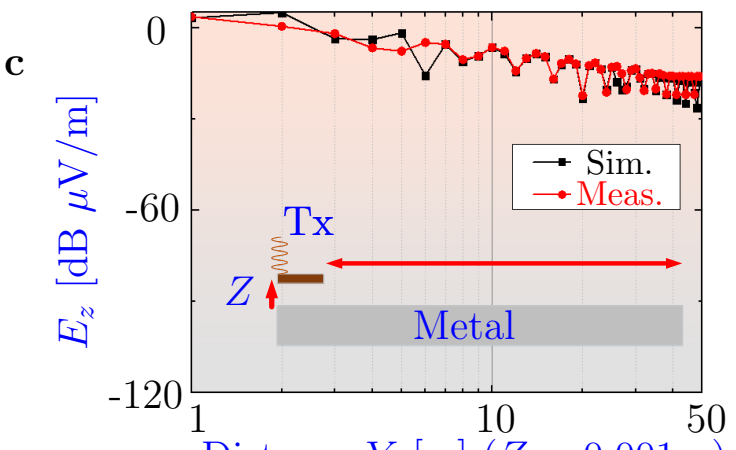

Distance, $Y[\mathrm{~m}](Z=0.001 \mathrm{~m})$

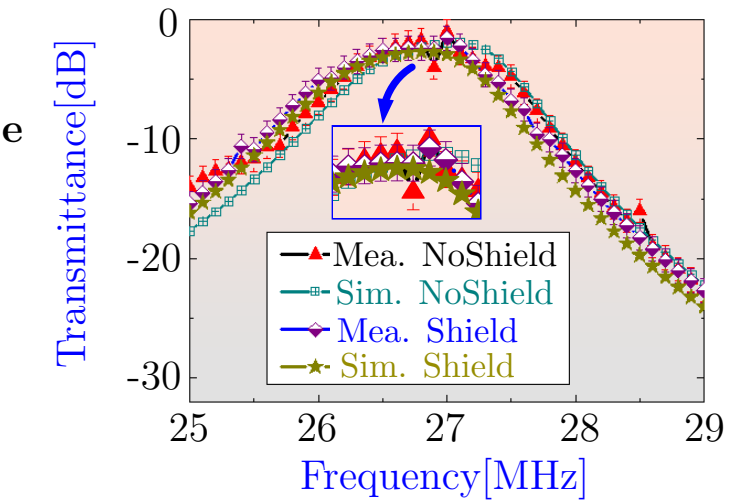

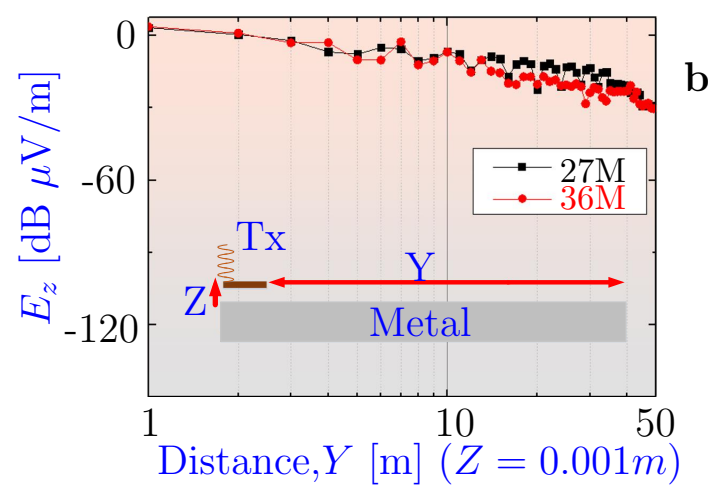
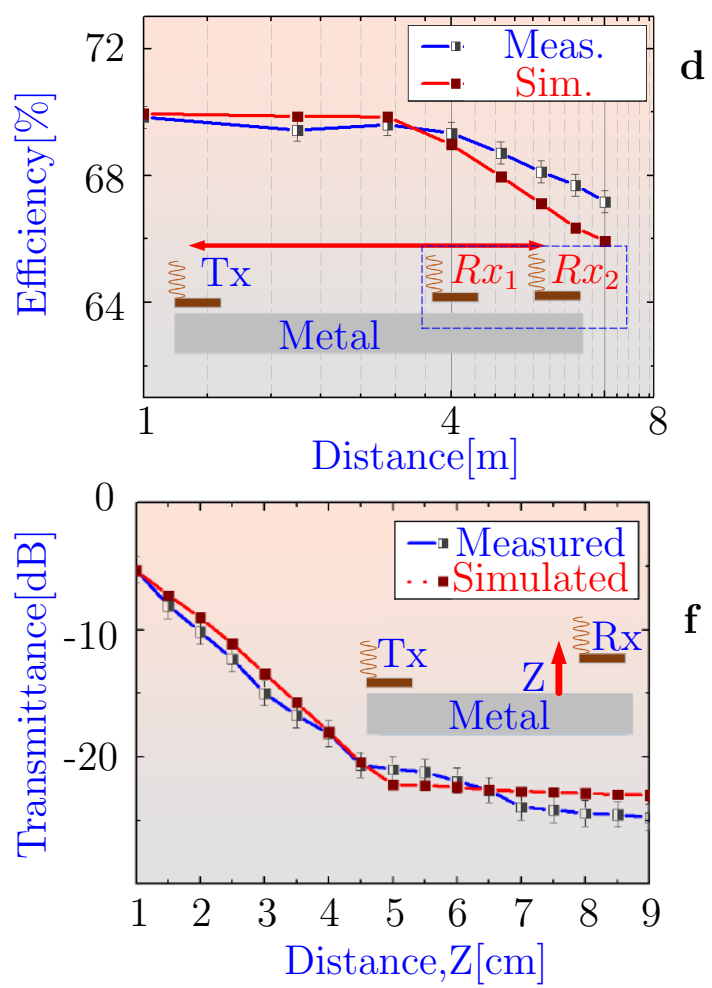

Fig. 2: Experiment and Simulation results: Zenneck Wave at metal-air interface (a) Ground Backed Impedance resonator system, with a half wavelength helical coil. (b) Experimental results of the $\mathrm{Z}$ component of the Electric field in the Y-direction 1 to 50 $m$, shows a slow attenuation rate. Two resonators with frequencies 27 and $36 \mathrm{MHz}$ were designed and compared. The resonators were placed at a vertical distance of $Z=$ $0.001 \mathrm{~m}$ above the metal surface.(c) Measured and simulated results comparison of E field attenuation along Y-direction at $27 \mathrm{MHz} ; \mathrm{Z}=0.001 \mathrm{~m}$. (d) Multi receiver power transfer efficiency. (e) Experimental and simulated results of the transmittance parameters, when transmitting and receiving unit are under shield and no-shield conditions. (f) Evanescent field decay experiment. 

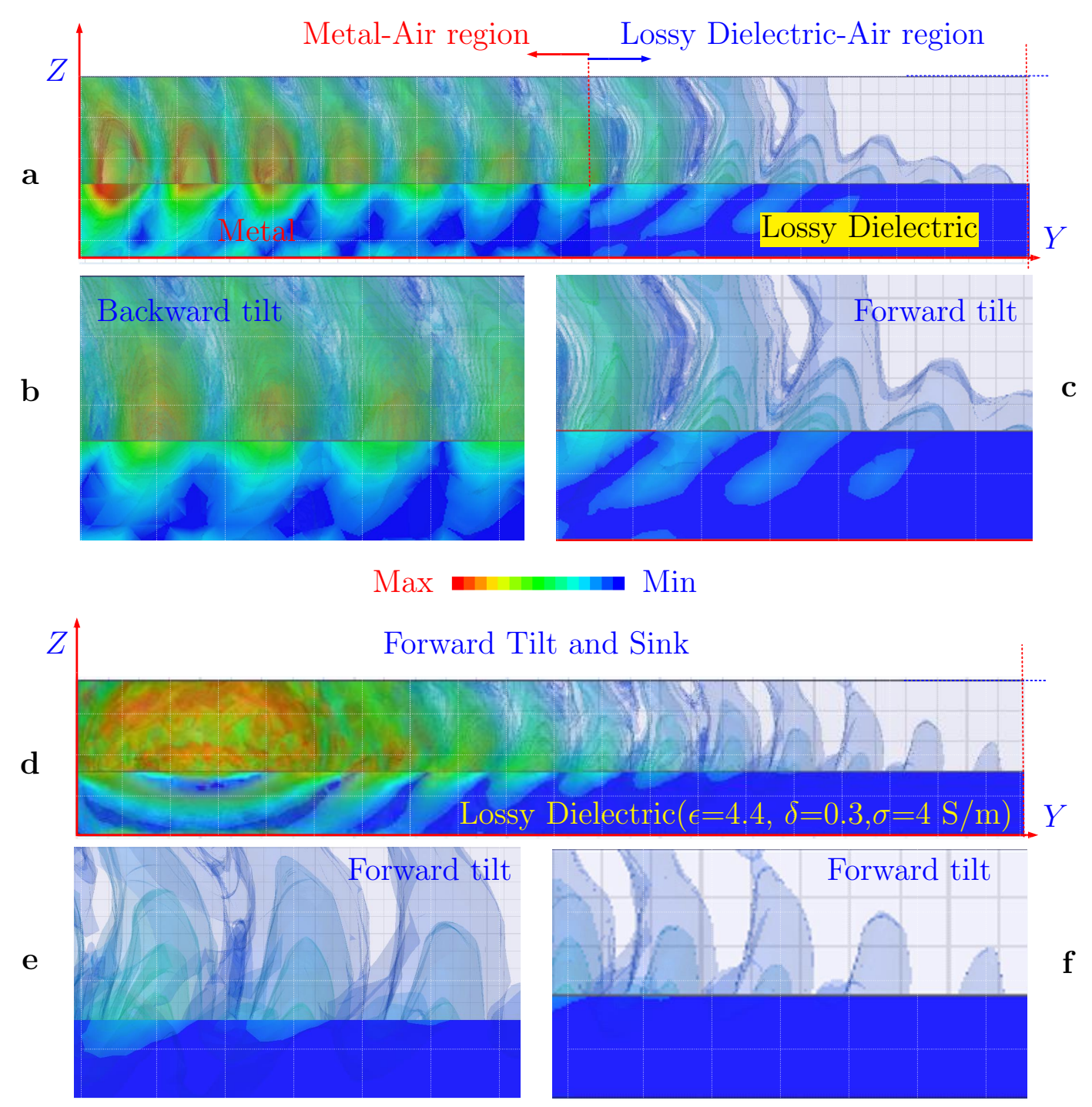

Fig. 3: Simulation of Iso-Phases (a) Half metal(Aluminum) and Half Lossy dielectric $(\epsilon=4.4, \delta=0.3, \sigma=4 \mathrm{~S} / \mathrm{m})$ (b) Inset View of Iso-Phases on metal (c) Inset view of Iso-phases in lossy dielectric. (d)Iso-Phases for lossy dielectric $(\epsilon=4.4, \delta=0.3, \sigma=4 \mathrm{~S} / \mathrm{m})$ (d) Inset view of Iso-Phases at the beginning of the lossy dielectric media (e) Sinking of the Iso-phases into the lossy dielectric 

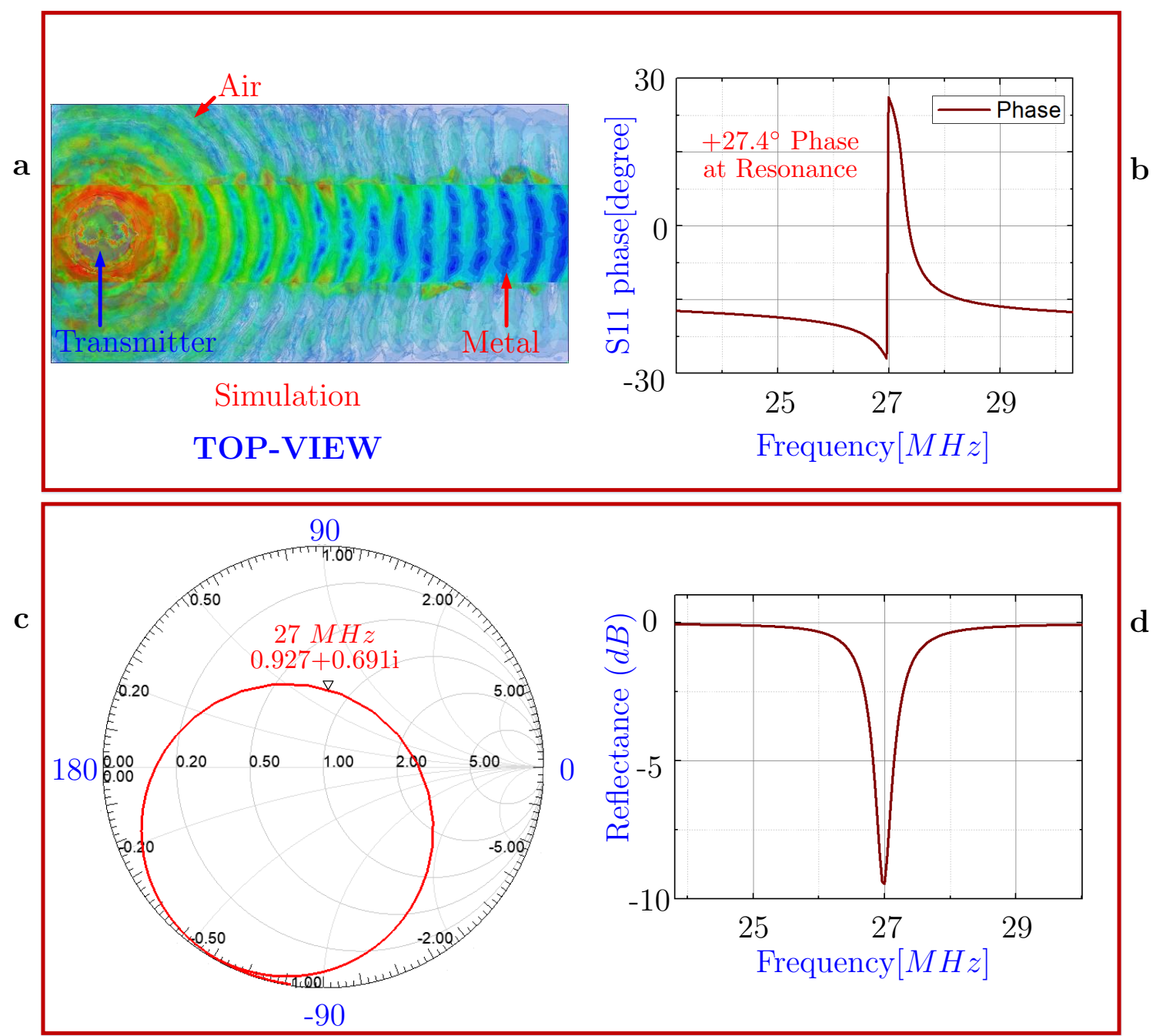

Fig. 4: Simulation (a)ANSYS HFSS simulation of the E-field modes(Top-View) (b) The HFSS simulation - S11 Phase angle at reosnance is positive, hence the ZW system is not a capacitive system. (c) HFSS simulation- Smith Chart of the S11 shows a positive imaginary quantity. Hence, inductive property is dominant (d)HFSS simulation- Reflectance at $27 \mathrm{MHz}$ resonance. 

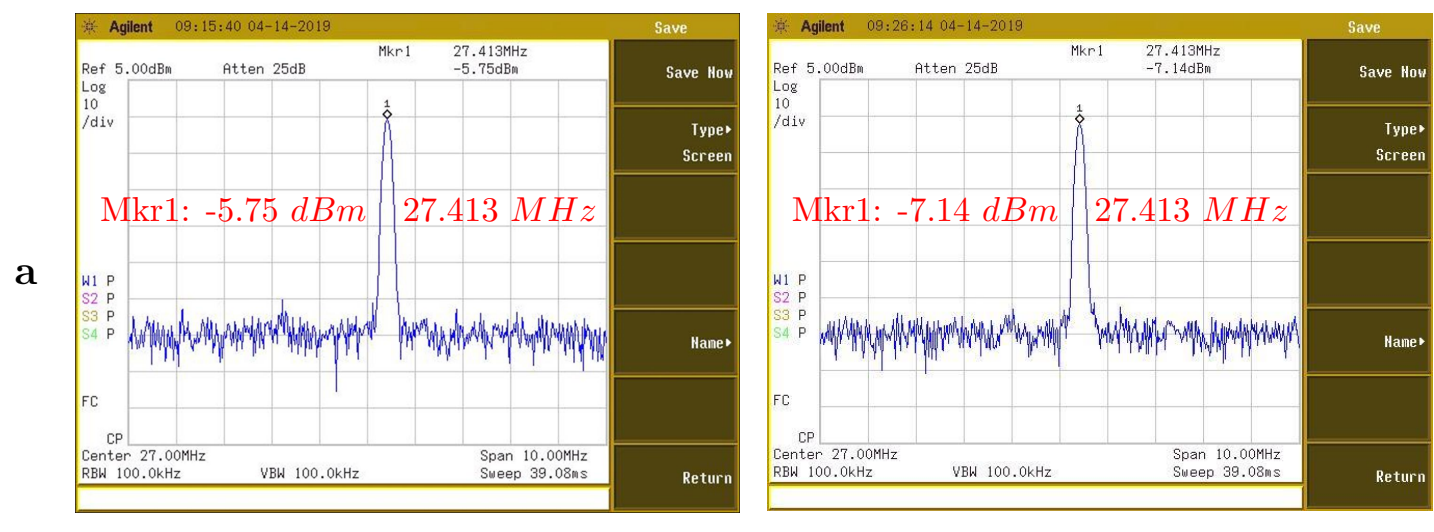

b
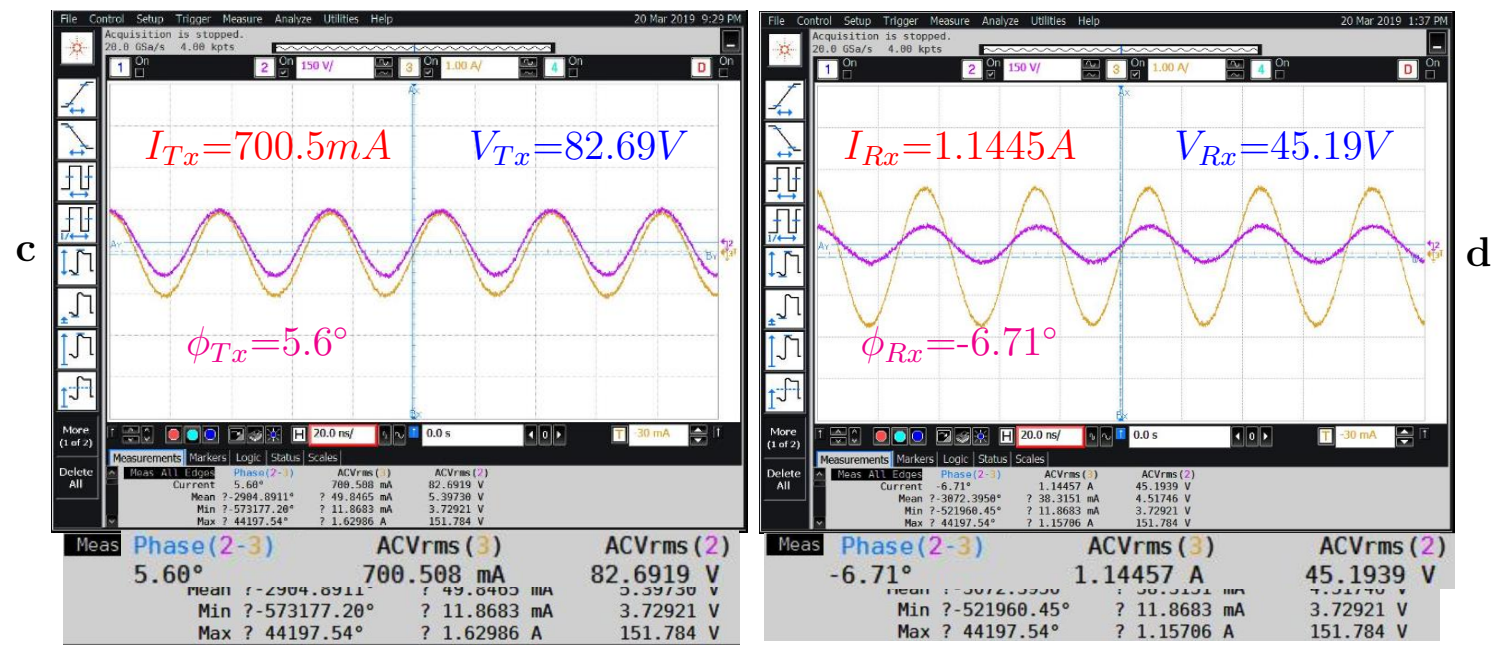

Measured Result

Fig. 5: Measurements (a) Spectrum analyzer measurement under open conditions (b) Spectrum analyzer measurement under shielding conditions. For spectrum analyzer, the distance between transmitter and receiver was at $15 \mathrm{~m}$. (c)AC RMS Current, Voltage and Phase across Transmitter terminals (d) AC RMS Current, Voltage and Phase across Receiver terminals. The distance between transmitter and receiver was at $8 \mathrm{~m}$. 
smart manufacturing floors, and secured shipping containers ${ }^{27-29}$.

\section{Results}

Please note, that the experimental setup is described in the section 1 of the supplementary material.

The key concept of this study is presented in Fig.1, the detailed analytical model and solution is presented in the supplementary material(under section: analytical formalism). The Fig. 1 a, shows the mechanism of Tesla transformer based wireless power transfer system. The primary coil consists of low number of turns, while the secondary has large number of turns(quarter-wave). One end of the secondary is left freely suspended in the air. Sometimes, a toroid is attached to the free end of the secondary to restrict the electric field buildup to prevent discharges. The primary and the secondary coils on both the transmission and the receiving end share the common ground. The generator, which operates as a high frequency AC source, is also grounded to the grid, which is in-turn grounded to the earth.

Approach followed in this study. The Fig. $1 \mathrm{~b}$ shows the schematic diagram of the system to excite zenneck waves at metal-air interface. Apart from exciting TM- waves using the GBI resonators at the metal-air interface, we use two critical concepts of Tesla transformer, namely- half wave helical coil (Tesla transformer uses a quarter wave coil), in the secondary to build high potential differences across the terminals of the resonator and grounding the coils to the grid ground, capacitively via the metal. This pulls the reference potential of the metal to the same level as the grid ground. Thus, metal is transformed into a neutral entity ${ }^{29}$. 
Half wave helical coil. It is well known that a quarter-wave open ended helical coil when mounted over a planar metal acts as a radiating antenna, notable application-vehicle mounted antennas, where the metal body provides a natural ground for the helical coil loaded antenna. In order to prevent radiation we used half wave helical coil. It is well known that, in the 3 to $40 \mathrm{MHz}$ range the reception parameters of an electrically small antenna needs improvement. In the supplementary material, the section "Electrical Length" describes the approach followed to address the above parameters in this study, in details. In the supplementary material(section 3) Sommerfeld analytical model is listed, the controversy surrounding the evaluation of the integral is mentioned. Finally, a commentary on the placement of pole as per the permissible Riemann sheet on the complex plane, in the case of metals is provided.

In the case of the proposed method, likelihood of the waves falling in the category of SP, SW and surface plasmon polariton (SPP) is eliminated when one considers the following conditions:

- SW:Corrugated metal structures are needed to increase the refractive index in order to excite SW's. Or an air-dielectric-metal(three layer) interface is needed. Alternatively, inductive surface impedance is needed to excite $\mathrm{SW}^{11,14,18,19,24}$.

- SPP and SP: Can not be excited at flat metal-air interfaces, without total internal reflection. Other methods of SPP excitation is based on grooves and near-field highly focused optical beams ${ }^{11,14,18,19,24}$.

Relation between SP and ZW. Interestingly, articles have appeared on relationship between SP and ZW. Most notably the article by Jeon et. al, where they mention in the title $\mathrm{THz}$ Zenneck surface wave and in brackets $\mathrm{THz}$ surface plasmon on metal sheets ${ }^{17}$. 
This arises from the fact that the pole and zero of the reflection coefficient $(R(\Lambda))$ in the Sommerfeld integral(equation S 30 and S31 in the supplementary material) on the permissible Riemann sheet coincide ${ }^{21}$. Sarkar et al. further note that the ZW attenuation rate along the interface is frequency independent, while attenuation rate in SP is frequency dependent ${ }^{21}$.

Equi-phases. The Fig. $1 \mathrm{c}$, shows the equi-phases generated due to the localized field oscillations on the metal-air interface. The phase velocity of the wave in the metal is faster than the free space, hence a backward tilt with an angle $\phi$ is observed, in accordance with ${ }^{9}$. The angle of tilt has been derived in this work from the 1907's article of Zenneck, which satisfy the Maxwell's boundary conditions:

$$
\phi_{0}=\sin ^{-1}\left[\left(\sqrt{\frac{\nu \sigma \epsilon_{0}}{\sigma^{2}+\nu^{2}\left(\epsilon^{\prime}-j \epsilon^{\prime \prime}\right)^{2}}}\right)^{-1}\right]
$$

Where, $\nu=2 \pi / \lambda ; \sigma$ is conductivity; complex permitivitty $\epsilon^{\prime}-j \epsilon^{\prime \prime}$; and free-space permitivitty $\epsilon_{0}$. The corresponding $\phi_{0}=90-\phi$, this was also mentioned in ${ }^{24}$. The derivation of the above equation is listed in the supplementary material(S33-S36). In case of metal-air interfaces the quantity $\phi_{0}$ becomes negative and hence a backward tilt. On the otherhand for air-lossy dielectric this angle is a positive quantity and hence a forward tilt.

Sinking of Equi-phases. Likewise, in the Fig. 1 d, the equi-phases undergo a forward tilt and subsequent sinking when they encounter a lossy dielectric ${ }^{16,24,25}$.

Hallmark of Zenneck waves. The ZW properties of the proposed system have been experimentally observed and are presented in Fig.2. The resonator system is shown in the Fig. 2 a, dimensions and parameters are presented in the supplementary material(Fig S 10 and table ST 2). 
a. Frequency independent slow attenuation rate. The Fig. 2 b, shows the comparison of the attenuation rate of the E-field $[d B \mu V / m]$ for the two resonator systems designed for operating frequencies of $27 \mathrm{MHz}$ and $36 \mathrm{MHz}$. The transmitter and receiver was fixed at a height of $Z=0.001 \mathrm{~m}$ above the metal surface. However, the receiver was moved along the interface ( $Y$-direction) and the corresponding values were recorded ${ }^{21}$. It is observed that the E-field values along the metal show a slow attenuation rate, independent of the frequency. This property is consistent with the ZW's as reported by Schelkunoff, Sarkar et al. and Barlow ${ }^{20-22,24}$. The Fig 2 c, shows the measured and simulated results of the attenuation rate at $27 \mathrm{MHz}$. The simulation was done using Ansys high-frequency structure simulator(HFSS). It is observed that the experiments and simulation model are in excellent agreement.

b. Multi receiver efficiency. The fig $2 \mathrm{~d}$, shows the multi receiver efficiency from $1 m$ to $8 m$ distance. Two receivers with identical loads were used of 20 watts each. The simulation result of the transmittance parameters are listed in the supplementary material (section 5). It is observed that the system efficiency varies between $66 \%$ to 62 $\%$ for a range of 1 to $8 \mathrm{~m}$. The power transfer metrics at $8 \mathrm{~m}$ and $15 \mathrm{~m}$ are listed in the supplementary material(tables ST 6 and ST 7)

c. Leaky or partial metal shields. The Fig. 2 e, shows the comparison of measured and simulated results of the transmittance parameters under leaky shielded and non-shielded conditions. The transmittance parameters were observed using the state-ofthe-art vector network analyzer. The FEM model is in good agreement with the measured results. It is observed that the proposed system, unlike the coupled WPT systems, has the ability to perform without any significant efficiency degradation ${ }^{2,26}$. 
d. Evanescent field/exponential decay. An exponential E-field decay is also observed in the normal direction away from the metal-air interface(listed in Fig. $2 \mathrm{f}$ ), consistent with the evanescent property of the ZW's ${ }^{9-11,16,20-25}$.

e. FEM Simulation model and Sinking of Equi-phases The equi-phases mentioned in the Fig. $1 \mathrm{c}$ and d, is shown in the Fig. 3 a to d. The simulation set up; in the Fig 3a two kind of materials are used comprising of equal lengths of metal and lossy dielectric. It is observed that the equi or Iso-phases are tilted backwards in the air, as long as metal-air interface exists as shown in Fig 3 a and b. Where as, for a case of Lossy dielectric-Air interface, the same Iso-Phases are tilted forwards in the air. When we look the simulation of lossy dielectric-air interface as shown in Fig. $3 \mathrm{~d}$ to $\mathrm{f}$; the Iso-phases are titled forwards in the air, in the direction of propagation. The Fig. 3 and in the supplementary material the fig S 16, confirms the implications of equation 1.

g. Eddy current effect. The current carried in the primary of the resonator coil, is effected by the eddy currents generated on the metal. This effect was reduced by increasing the spacing between the coil and the GBI resonator from $105 \mathrm{~mm}$ to $260 \mathrm{~mm}$.

h. Attenuation rate along different interfaces. If the proposed method is exciting Zenneck waves at the metal-air interface, then, they must also show similar properties across various other conductive media. The Fig. S5, shows the attenuation characteristics across aluminium (conductivity, $\sigma=3.8 \times 10^{7} \mathrm{~S} / \mathrm{m}$ ), iron $\left(\sigma=1.03 \times 10^{7} \mathrm{~S} / \mathrm{m}\right.$ ) and seawater $(\sigma=4 S / m$ and $\epsilon=81)$. It is observed that the attenuation rate in seawater is faster than metal. 
Additional commentary. Recently, the Sommerfeld-Zenneck wave behaviour has been demonstrated in the centimeter range ${ }^{30}$. Interestingly, (the fig $2 \mathrm{C}$ top-view of $^{30}$ ) of the article shows the simulation of the E-field modes identical to the Fig. 4 a. The result listed in Fig.4 a is in the $M H z$ range. At a first glance, the presented ZW system looks like a capacitive power transfer system. But, this is misleading, we need to look at the details of the phase of the reflectance parameters, when the transmitter is placed in the proximity of the metal. Fig. $4 \mathrm{~b}$ shows the phase angle at resonance to be positive. Moreover, the Fig. $4 \mathrm{c}$ shows the smith chart results, which shows a positive value for the complex quantity of the impedance at resonance. Finally, the reflectance parameters magnitude in $d B$ is shown to have a resonance at $27 \mathrm{MHz}$. The corresponding measurement results are shown in the supplementary material Fig S17. Ofcourse, all these observations are made by placing the transmitter on the metal, with an insulator. Based on the results of Fig. $4 \mathrm{~b}$ to $\mathrm{c}$ and Fig S17, it can be concluded that the ZW system is not a capacitive power transfer system ${ }^{31,32}$.

Power Transfer Metrics. The Fig.5a and b shows the received power under open and leaky shielded conditions, the measurement is performed using spectrum analyzer on the receiver side. The transmitter is fed with $0 \mathrm{dBm}$ from a spectrum analyzer, the sweep conditions are start frequency $22 \mathrm{MHz}$, center frequency $27 \mathrm{MHz}$ and the stop frequency is $32 \mathrm{MHz}$. The distance between transmitter and receiver is $15 \mathrm{~m}$. The Fig. 5 $\mathrm{c}$ and $\mathrm{d}$ show the measured AC RMS current, voltage and phase values across transmitter and receiver, transmission range is 8 meters. Interestingly, the transmitter to receiver efficiency is high; $\eta=\left[V_{R x_{R M S}} \times I_{R x_{R M S}} \times \cos \phi_{R x} / V_{T x_{R M S}} \times I_{T x_{R M S}} \times \cos \phi_{T x}\right] \times 100=$ $[45.19 \times 1.1445 \times \cos (-6.71) / 82.85 \times 0.7 \times \cos (5.68)] \times 100=89.1 \%$. However, when one calculates the power amplifier to receiver efficiency, the figure drops to $64 \%$. The input 
from signal generator is $-4 \mathrm{dBm}$, the power amplifier [the model Prana DP 300] used has a gain of $53 \mathrm{dBm}$. Therefore, the total power fed into transmitter is $49 \mathrm{dBm}$, which is 79.4 Watts. There are reflection losses at the transmitter end. Use of an appropriate impedance matching network on the load end can improve the overall efficiency, i.e. power amplifier to the receiver load. In the supplementary material the measurement for the system under leaky metal shield is listed in Fig.S 18. The transmitter to receiver power transfer efficiency is $82.29 \%$; the power amplifier to receiver efficiency is $59.7 \%$.

\section{Discussions}

We have demonstrated the excitation of waves on metal surfaces that can be used for delivering electrical power to multiple devices. The waves show slow attenuation property similar to ZW's along the metal-air interface and can be used for efficient powering of devices up to 8 meters using this arrangement. We also show that ZW's can be used for transmitting power across partial metal enclosures. Therefore, the resonator system has the ability to overcome electromagnetic shielding and can be used for powering devices under leaky metal enclosures since the excited waves are non-radiative in nature. Power transmission to multiple receiving resonators with uniform efficiency has also been established experimentally and shows excellent agreement with simulation. The simulation result is compared with coupled mode power transfer system in the supplementary(Fig. S10). Existing coupled mode WPT systems undergo peak splitting when multiple receiving units are involved. Our study shows that using a wave-based mode of transmission, we can solve this issue. The efficiency of power transmission increases when multiple receiving units are present, as the power is uniformly spread across the metal surface. This kind of increase, due to multiple receiving units was also observed in a widely followed article, where weakly coupled WPT system is used ${ }^{33}$. The maximum value of $\mathrm{E}$ 
and H-field emitted by the system is $34 \%$ and $89 \%$ lower than the permitted values, regulated by the ICNIRP guidelines at this frequency(Supplementary material Fig. S 12, S 13 and Table ST 3). Thus, this system should not pose as an occupational hazard to human operators. The proposed system has no effect on other devices operating in vicinity(supplementary material demo video links). Since ZW, SW, SP and SPP have an evanescent field, the transceivers need to be in proximity to the interface, on the other hand free space wave bases systems do not have this limitation. However, most free-space wave systems have limitations in power handling, efficiency and can not perform in the presence of leaky shielded environments.

\section{References}

1. Tesla,N., U.S. patent 1,119,732 (1914).

2. Kurs, A., Karalis, A., Moffatt, R., Joannopoulos, J. D., Fisher, P., Soljačić, M. Wireless Power Transfer via Strongly Coupled Magnetic Resonances. Science 317, 83-86 (2007).

3. Pan, K., Fan, Y., Leng, T., Li, J., Xin, Z., Zhang, J., Hao, L., Gallop, J., Novoselov, K. S. \& Hu, Z. Sustainable production of highly conductive multilayer graphene ink for wireless connectivity and IoT applications. Nature Comm. 9,5197 (2018).

4. Dasgupta, A., Mennemanteuil, M.M., Buret, M., Cazier, N., Colas-des-Francs, G., \& Bouhelier, A. Optical wireless link between a nanoscale antenna and a transducing rectenna. Nature Comm. 9, 1992(2018).

5. Folcher, M., Oesterle, S., Zwicky, K., Thekkottil, T., Heymoz, J., Hohmann, M., Christen, M., Daoud El-Baba, M., Buchmann, P., \&Fussenegger, M. Mind-controlled trans- 
gene expression by a wireless-powered optogenetic designer cell implant. Nature Comm. $5,5392(2014)$.

6. Assawaworrarit, S., Yu, X., \& Fan, S. Robust wireless power transfer using a nonlinear paritytime-symmetric circuit. Nature 546, 387-390 (2017).

7. Nguyen, H., \& Agbinya,J. I. Splitting Frequency Diversity in Wireless Power Transmission. IEEE Trans. Power Electronics 30, 6088-6096(2015).

8. Stevens, C.J., Magnetoinductive Waves and Wireless Power Transfer,IEEE Transactions on Power Electronics 30, 6182 (Nov. 2015.)

9. Zenneck, J. Über die Fortpflanzung ebener elektromagnetischer Wellen längs einer ebenen Leiterfläche und ihre Beziehung zur drahtlosen Telegraphie. Ann. d. Phys. 23, 846-866 (1907).

10. Sommerfeld, A. N. Über die Ausbreitung der Wellen in der drahtlosen Telegraphie. Ann. d. Phys. 28, 665-736(1909).

11. Lereu, A.L., Passian, A., Thundat, T.,\& Ferrell, T. L. Optical modulation processes in thin films based on thermal effects of surface plasmons. Appl. Phys. Lett., 86, 154101 (2005).

12. Hill, D. A., \& Wait, J. R. Excitation of the Zenneck surface wave by a vertical aperture. Radio Science 13, 969 (1978).

13. Anh, N. D., Chun,B.J., Choi, S.,Kim, D.E., Kim,S., \& Kim,Y.J. Plasmonic dynamics measured with frequency-comb-referenced phase spectroscopy. Nature Physics,(Nov. 2018). 
14. Lee, J., Tymchenko, M., Argyropoulos, C., Chen, P.Y., Lu, F., Demmerle,F., Boehm, G., Amann,M.C., Alu, A., \& Belkin, M.A. Giant nonlinear response from plasmonic metasurfaces coupled to intersubband transitions. Nature 511,65-69(2014).

15. Maier, S. Clear for launch. Nature Physics 3,301-303(2007).

16. Jangal,F., Bourey, N., Darces, M., Issac, F. \& Hélier, M. Observation of Zenneck-Like Waves over a Metasurface Designed for Launching HF Radar Surface Wave. Hindawi, International J. of Antennas and Propagation 2016,1 (2016).

17. Jeon,T.I., Zhang, J., \& Grischkowsky, D. THz Zenneck surface wave (THz surface plasmon) propagation on a metal sheet. Appl. Phys. Lett. 86, 161904 (2005).

18. Hibbins, A.P., Evans, B. R., \& Sambles, J. R. Experimental Verification of Designer Surface Plasmons. Science 308, 670-672 (2005).

19. Goubau,G. Surface Waves and Their Application to Transmission Lines. Journal of Appl. Physics 21, 1119(1950).

20. Schelkunoff, S. Anatomy of "Surface waves". IRE Trans. on Antenna and Propagation 7, 133-139 (1959).

21. Sarkar,T. K., Abdallah, M. N., Salazar-Palma, M., \& Dyab, W. M. Surface PlasmonsPolaritons, Surface Waves, and Zenneck Waves: Clarification of the terms and a description of the concepts and their evolution. IEEE Antennas and Propagation Magazine 59, 77-93(2017).

22. Sarkar,T.K., Dyab,W.M., Abdallah, M.N.,Salazar-Palma, M., Prasad, M.V.S.N., \& Ting, S. Application of the Schelkunoff formulation to the Sommerfeld problem of a 
vertical electric dipole radiating over an imperfect ground. IEEE Trans. on Antennas and Propagation 62, 4162-4170 (2014).

23. Corum, J.F., \& Corum, K.L. U.S. Patent US9912031B2 (2018).

24. Barlow, H.M. \& Cullen, A.L. Surface Waves. Proceed. of the IEE - Part III: Radio and Comm. Engineering 100, 329-341 (1953).

25. Ling, R. T., Scholler, J. D.,\& Ufimtsev, P. Ya. The Propagation and Excitation of surface waves in an Absorbing Layer. Progress In Electromagnetics Research 19, 4991(1998).

26. Li, J., Huang, X., Chen, C., Tan, L., Wang W. and Guo,J. Effect of metal shielding on a wireless power transfer system. AIP Advances 7, 056675 (2017).

27. Bien, F., Oruganti, S.K., Heo, S.H., Ma,H., \& Seo, S. PCT/KR2016/008396 (2016).

28. Oruganti, S. K., Kaiyrakhmet, O. \& Bien, F., Wireless power and data transfer system for internet of things over metal walls and metal shielded environments. URSI Asia-Pacific Radio Science Conference, 318(2016).

29. Van Neste, C.W., Hull, R., Hawk, J.E., Phani A., \& Thundat, T. Electrical excitation of the local earth for resonant, wireless energy transfer. Cambridge Wireless Power Journal, 3, 117-125(2016).

30. Sergeichev,K.F., Karfidov,D.M., Andreev, S. E., Sizov, Yu. E., \& Zhukov, V.I. Excitation and Propagation of SommerfeldZenneck Surface Waves on a Conducting Strip in the Centimeter-Wave Band. Journal of Comm. Tech. and Electronics, 63,326,2018. 
31. Fernandes,R. D., Matos J. N. \& Carvalho,N. B., Resonant Electrical Coupling: Circuit Model and First Experimental Results, IEEE Transactions on Microwave Theory and Techniques, 63, 2983, 2015.

32. Lu, F., Zhang, H. \& Mi, C., A Review on the Recent Development of Capacitive Wireless Power Transfer Technology, Energies, 10,1752, 2017.

33. Kurs, A., Moffatt, R., \& Soljačić, M. Simultaneous mid-range power transfer to multiple devices. App. Phys. Lett., 96, 044102-3(2010). 